\title{
含吗啉环的 2-苯并咪唑基氰基甲酮肟醚的合成及杀菌活性
}

\author{
曲智强李付博邢程远 姜 林* \\ (山东农业大学化学与材料科学学院 泰安 271018)
}

\begin{abstract}
摘要 利用活性结构拼接原理, 以 2-苯并咪唑乙腈、亚硝酸钠、氯乙酰吗啉、卤代烃等为原料, 经过肟化、取代和醚 化等三步反应合成了一系列新型的[1-(4-吗啉基)羰基亚甲基苯并咪唑-2-基]氰基甲酮肟醚(4a $\sim \mathbf{4 h}$ ), 其结构经 IR, NMR, MS 和元素分析确认. 初步生物活性测试结果表明, 部分化合物对生菜灰霉菌和番茄菌核菌有较高的抑制活性, 与对照 药剂多菌灵相比，含 4-氟苄基或 4-氯苄基的化合物 4e, 4f 活性优异.

关键词 肜醚; 苯并咪唑; 吗啉; 杀菌活性
\end{abstract}

\section{Synthesis and Antifungal Activity of Novel Benzimidazol-2- ylcyanoketone Oxime Ethers Containing Morpholine Moiety}

\author{
Qu, Zhiqiang Li, Fubo Xing, Chengyuan Jiang, Lin* \\ (College of Chemistry and Material Science, Shandong Agricultural University, Taian 271018)
}

\begin{abstract}
A series of benzimidazol-2-ylcyanoketone oxime ethers containing morpholine moiety $(\mathbf{4 a} \sim \mathbf{4 h})$ were synthesized from 2-benzimidazolyl acetonitrile, sodium nitrite, chloroacetyl morpholine and haloalkane/benzyl chloride via sequential reactions of oximation, substitution and etherification. The structures of the target compounds were elucidated by IR, NMR, MS, and elemental analysis. All the target compounds were tested for in vitro antifungal activities against Botrytis cinerea and Sclerotinia sclerotiorum by the mycelium growth rate test, and the results indicated that some compounds displayed higher antifungal activity as compared to carbendazim, among which the compounds containing 4-fluorobenzyl or 4-chlorobenzyl moiety (4e, 4f) have excellent activity.
\end{abstract}

Keywords oxime ether; benzimidazole; morpholine; antifungal activity

肜醚类化合物具有广泛的生物活性, 如杀虫 ${ }^{[1]}$ 、杀

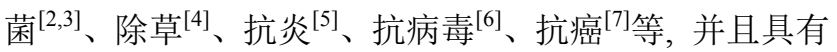
高效、低毒等优点, 因此, 该类化合物的分子设计、合 成及生物活性研究成为农药和医药创制的热点之一. 自 从 20 世纪 70 年代开发出第一个肜醚类杀菌剂-霜嫝氰 以来, 又成功开发出一些高效的该类杀菌剂, 如啶斑 肜、醚菌酯、肜醚菌胺等 ${ }^{[8]}$. 近年来, 湖南化工研究院开 展了许多具有杀虫、杀菌活性的肜醚类化合物的设计与 合成研究 ${ }^{[9,10]}$, 获得了具有杀螨、杀菌活性的候选农药 品种 HNPC-A3066; 上海农药所也创制出苯乙酮肜醚类 杀菌剂氯苯肜唑 ${ }^{[11]}$. 另一方面, 许多含苯并咪唑结构或 吗啉结构的化合物也具有非常好的杀菌活性, 如多菌 灵、苯菌灵、烯酰吗啉、丁吡吗啉等 ${ }^{[12,13]}$. 受以上研究 成果的启示, 结合我们已开展的有关肟醚和苯并咪唑类
化合物的合成与生物活性研究 ${ }^{[14,15]}$, 本文利用活性结构 拼接法, 将肟醚和乙酰基吗啉结构引入苯并咪唑母环 中, 设计合成了一系列未见文献报道的 [1-(4-吗啉基)羰 基亚甲基苯并咪唑-2-基]氧基甲酮肜醚 $(\mathbf{4 a} \sim \mathbf{4 h})$, 并测 试了化合物对两种植物病原菌的离体杀菌活性. 目标化 合物的合成路线见 Scheme 1.

\section{1 结果与讨论}

\section{1 化合物合成}

中间体 [1-(4-吗啉基)羰基亚甲基苯并咪唑-2-基]氰 基甲酮肜(3)的制备中, 曾用氢氧化钠代替无水碳酸钾 作缚酸剂, TLC 检测显示有多个点, 表明有副产物生成. 这可能是氢氧化钠碱性太强, 也使弜羟基转化为负离

\footnotetext{
*E-mail: jiangl@sdau.edu.cn

Received April 13, 2015; revised May 29, 2015; published online June 6, 2015.

Project supported by the Science and Technology Development Plan of Shandong Province (No. ZR2013GZX20109).

山东省科技发展计划(No. 2013GZX20109)资助项目.
} 


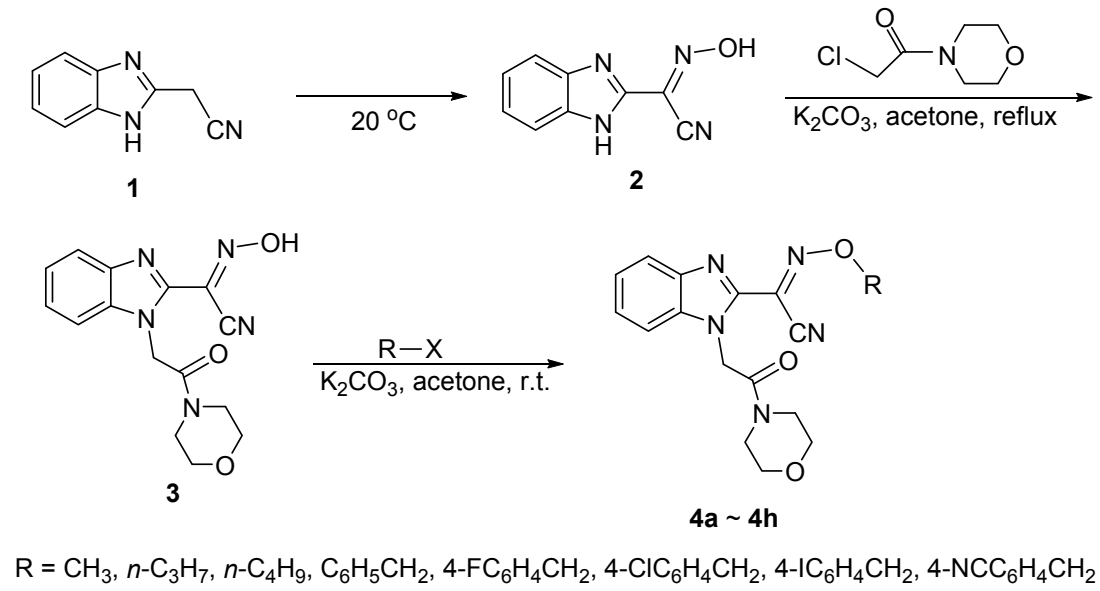

图式 1 化合物的合成路线

Scheme 1 Synthetic route of the target compounds

子, 在与氯乙酰吗啉反应时还发生了 $O$-烷基化反应, 生 成副产物. 目标化合物 $\mathbf{4 b} \sim \mathbf{4 h}$ 的合成中, 反应体系中 加入了碘化钾, 可使反应物澳代烷或茮基氯在丙酮溶液 中转化为活性高的碘代烷或苄基碘, 从而使反应速率加 快, 一般 1 3 h 即可完成反应. 如果不加碘化钾, 反应 时间则需 $5 \mathrm{~h}$ 左右. 反应中我们也尝试用氢氧化钠替代 无水碳酸钾作碱催化剂, 但收率较低( $30 \%$ 左右). 化合 物的结构经 IR, ${ }^{1} \mathrm{H}$ NMR, MS 和元素分析确认, 部分化 合物(4a、4d) 还经 ${ }^{13} \mathrm{C}$ NMR 确认.

\section{2 波谱解析}

目标化合物 ${ }^{1} \mathrm{H} \mathrm{NMR}$ 中, 吗啉环上的 $\mathrm{NCH}_{2}$ 质子为 两组峰, 分别在 $\delta 3.30 \sim 3.57$ 和 $3.53 \sim 3.65$ 处, 与未取代 的吗啉环相比 $(\delta=2.72)$, 其化学位移值明显地向低场移 动, 这与文献报道 ${ }^{[16]}$ 的含酰基吗啉结构的化合物情况 相近. 吗啉环 $\mathrm{OCH}_{2}$ 质子为一组多重峰, 在 $\delta 3.59 \sim 3.74$ 处, 与未取代的吗啉环质子差别较小 $(\delta=3.52)$. 肜醚键 的 $\mathrm{CH}_{2}$ 受电负性大的 $\mathrm{O}$ 原子吸电子影响, 化学位移较 大，当该亚甲基与烷基相连时 $(\mathbf{4 b}, \mathbf{4 c}), \delta$ 在 $4.38 \sim 4.42$ 处; 当它与苯基相连时 $(\mathbf{4 d} \sim \mathbf{4 h})$, 质子还受到苯环去屏 蔽效应的影响, 化学位移更向低场移动, $\delta$ 在 $5.60 \sim 5.74$ 之间. 此外, 位于苯并咪唑-2-基和酰基吗啉环之间的 $\mathrm{CH}_{2}$ 质子 $\delta$ 在 $4.89 \sim 5.31$ 之间.

在 IR 谱中, $\mathrm{C} \equiv \mathrm{N}$ 伸缩振动的弱吸收峰在 2225 $2238 \mathrm{~cm}^{-1}$ 处, 羰基的强伸缩振动吸收峰位于 1657 $1669 \mathrm{~cm}^{-1}$, 吗啉环 $\mathrm{C}-\mathrm{O}-\mathrm{C}$ 吸收峰在 $1115 \mathrm{~cm}^{-1}$ 附近, 1024 1032 $\mathrm{cm}^{-1}$ 的强吸收峰可能是肜醚键 $\mathrm{C}-\mathrm{O}-\mathrm{N}$ 的伸缩振动吸收峰 ${ }^{[17]}$.

肜醚分子中因含有 $\mathrm{C}=\mathrm{N}$ 双键, 常常会存在 $Z 、 E-$ 式两种构型 ${ }^{[4,18]}$. 通过对目标化合物的 ${ }^{1} \mathrm{H}$ NMR 分析, 我 们注意到本文所合成的肜醚只有一种构型 $(E$-式), 其原 因可能是在 $4 a \sim 4 h$ 分子中, 肜醚结构 $\mathrm{C}=\mathrm{N}$ 键的碳原
子上一端连有体积很大的取代苯并咪唑基、另一端连有 体积小的氧基，故肜醚键上的烷/苄氧基位于氧基的同 一侧, 即倾向于热力学上较稳定的 $E$-式构型存在.

\section{3 抑菌活性}

目标化合物 $\mathbf{4 a}, \mathbf{4 d} \sim \mathbf{4 h}$ 对生菜灰霉菌有良好的抑 制活性，其中 $\mathbf{4 e}$ 和 $\mathbf{4 f}$ 活性最高, $\mathrm{EC}_{50}$ 分别为 $10.78 、 11.14$ $\mu \mathrm{g} / \mathrm{mL}$ ，但低于对照药剂多菌灵 $\left(\mathrm{EC}_{50} 18.71 \mu \mathrm{g} / \mathrm{mL}\right)$. 除 $4 \mathrm{c}$ 外，其余 7 种化合物对番茄菌核菌具有较高的抑制活 性, $\mathrm{EC}_{50}$ 在 $10.46 \sim 37.81 \mu \mathrm{g} / \mathrm{mL}$, 均低于多菌灵 $\left(\mathrm{EC}_{50}\right.$ $41.02 \mu \mathrm{g} / \mathrm{mL}$ ), 其中 $\mathbf{4 e}$ 和 $\mathbf{4 f}$ 有优异的杀菌活性, $\mathrm{EC}_{50}$ 分 别为 $10.82 、 10.46 \mu \mathrm{g} / \mathrm{mL} ; \mathbf{4 a}$ 和 $\mathbf{4 d}$ 也有比较强的杀菌活 性, $\mathrm{EC}_{50}$ 分别为 $25.76 、 19.38 \mu \mathrm{g} / \mathrm{mL}$.

从目标化合物结构和杀菌活性的关系可以看出(表 1), 当豚醚键的烃基为烷基时, 含甲基的化合物 $4 \mathrm{a}$ 的活 性优于其他化合物 $4 b, 4 c$, 说明烷基增大不利于提高活 性. 当烃基为取代苄基时, 苯环上含氟、氯原子的化合 物 $4 \mathrm{e}, \mathbf{4 f}$ 活性明显高于苯环未取代或苯环上含其他取代 基的化合物，即目标化合物的分子中，苯环上氟原子或 氯原子的引入提高了杀菌效果. 总体来看, 肟醚键烃基 为取代苄基的化合物，其活性优于烃基为烷基的化合 物.

\section{2 结论}

本文以 2-苯并咪唑乙腈、亚硝酸钠、氯乙酰吗啉和 卤代烃等为原料, 经过肜化、取代和醚化等三步反应合 成了 8 种新型的[1-(4-吗啉基)羰基亚甲基苯并咪唑-2基]氧基甲酮肟醚 $(\mathbf{4 a} \sim \mathbf{4 h})$. 用生长速率法测试了目标化 合物对生菜灰霉菌和番茄菌核菌的离体杀菌活性, 结果 表明部分化合物对两种植物病原菌有较高的抑制活性, 其中 $4 \mathrm{e}$ 和 $4 \mathrm{f}$ 表现出优异的活性, 高于对照药剂多菌灵, 其 $\mathrm{EC}_{50}$ (95\%置信限)在 $10.46 \sim 11.14 \mu \mathrm{g} / \mathrm{mL}$ 之间. 初步 
表 1 目标化合物 $\mathbf{4 a} \sim \mathbf{4 h}$ 的杀菌活性 $\left(\mathrm{EC}_{50}\right)$

Table 1 Antifungal activity as inhibition rate for the target compounds $\mathbf{4 a} \sim \mathbf{4 h}$

\begin{tabular}{|c|c|c|}
\hline \multirow{2}{*}{ Compd. } & \multicolumn{2}{|c|}{$\mathrm{EC}_{50} /\left(\mu \mathrm{g} \cdot \mathrm{mL}^{-1}\right)$} \\
\hline & Botrytis cinerea & Sclerotinia sclerotiorum \\
\hline $4 \mathbf{a}$ & $28.24(22.71 \sim 35.12)$ & $25.76(24.08 \sim 27.55)$ \\
\hline $4 b$ & $38.26(34.55 \sim 42.36)$ & $33.27(30.36 \sim 36.45)$ \\
\hline $4 c$ & $32.88(26.94 \sim 40.11)$ & $73.44(61.86 \sim 87.18)$ \\
\hline 4d & $25.34(20.22 \sim 31.75)$ & $19.38(17.82 \sim 21.08)$ \\
\hline $4 e$ & $10.78(8.71 \sim 13.34)$ & $10.82(8.50 \sim 13.77)$ \\
\hline $4 f$ & $11.14(8.75 \sim 14.17)$ & $10.46(8.45 \sim 12.94)$ \\
\hline $4 g$ & $25.30(20.13 \sim 31.79)$ & $37.81(34.49 \sim 41.45)$ \\
\hline $4 h$ & $24.00(22.76 \sim 25.31)$ & $24.42(20.93 \sim 28.47)$ \\
\hline Carbendazim & $18.71(13.98 \sim 25.04)$ & $41.02(32.85 \sim 51.21)$ \\
\hline
\end{tabular}

构效关系分析显示，该类化合物分子中肟醚键的烃基为 甲基、4-氯苄基、4-氟苄基时，杀菌活性高于结构类似 的其他化合物.

\section{3 实验部分}

\section{1 仪器与试剂}

北京泰克仪器有限公司 X-5 精密显微熔点测定仪 (温度计未校正); 美国 Nicolet 380 型傅立叶变换红外光 谱仪( $\mathrm{KBr}$ 压片); 美国 Mercury Plus-400 型核磁共振仪 $\left(\mathrm{CDCl}_{3}\right.$ 为溶剂, $\mathrm{TMS}$ 为内标 $)$; 美国 Agilent 1200 型液质 连用仪; 德国 Elementar 公司 Vario EL III 型元素分析仪; 宁波江南仪器厂 GXZ 型智能光照培养箱.

\section{2 实验方法}

\section{2 .1 化合物的合成}

2-苯并咪唑乙腈(1)由邻苯二胺和氰基乙酸乙酯为 原料按文献[19]方法制备, 苯并咪唑基氰基甲酮肜(2)参 考文献[20]合成.

[1-(4-吗啉基)羰基亚甲基苯并咪唑-2-基]氧基甲酮

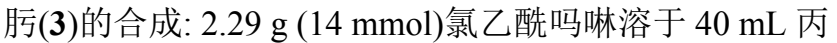

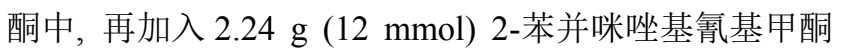
肜(2)和 $1.93 \mathrm{~g}(14 \mathrm{mmol})$ 无水 $\mathrm{K}_{2} \mathrm{CO}_{3}$, 加热回流 $3 \mathrm{~h}$, TLC 跟踪至反应完全 $[V$ (丙酠 $): V($ 石油醚 $)=1: 1]$. 冷却至 室温, 抽滤, 水洗、干燥得黄色固体; 滤液旋蒸浓缩后倒 入水中, 析出棕色固体. 合并粗产物, 用热乙醇洗, 得 黄色固体 $2.4 \mathrm{~g}$, 产率 $63 \%$. m.p. $225.8 \sim 227.1{ }^{\circ} \mathrm{C} ;{ }^{1} \mathrm{H}$ NMR $\left(\mathrm{CDCl}_{3}, 400 \mathrm{MHz}\right) \delta: 3.48 \sim 3.53(\mathrm{~m}, 4 \mathrm{H}, \mathrm{Morp}-\mathrm{H})$, $3.54 \sim 3.66(\mathrm{~m}, 4 \mathrm{H}$, Morp- $\mathrm{H}), 5.29$ [s, $2 \mathrm{H}, \mathrm{NCH}_{2} \mathrm{C}(=\mathrm{O})$ ], $7.28 \sim 7.74$ (m, 4H, Ar-H), 13.22 (s, 1H, OH); IR (KBr) v: $2238,1660,1589,1240,1105,1031 \mathrm{~cm}^{-1}$. Anal. calcd for $\mathrm{C}_{15} \mathrm{H}_{15} \mathrm{~N}_{5} \mathrm{O}_{3}$ : C 57.50, H 4.83, N 22.35; found C 57.16, H 5.08, N 22.04.

[1-(4-吗啉基)羰基亚甲基苯并咪唑-2-基]氰基甲酮

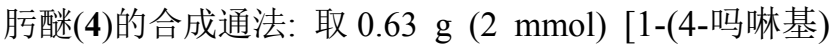

羰基亚甲基苯并咪唑-2-基]氧基甲酮肜(3)， $0.56 \mathrm{~g}$ (4 $\mathrm{mmol})$ 无水 $\mathrm{K}_{2} \mathrm{CO}_{3}$ 和 $0.34 \mathrm{~g}(2 \mathrm{mmol}) \mathrm{KI}$, 加至 $50 \mathrm{~mL}$ 二 颈烧瓶中(注: 合成中不加 $\mathrm{KI}$ ), 再加入 $10 \mathrm{~mL}$ 丙酮, 室 温摚拌均匀后于 $30 \mathrm{~min}$ 内滴加 $10 \mathrm{~mL}$ 含 $2.4 \mathrm{mmol}$ 碘甲 烷、溴代烷或取代苄基氯的丙酥溶液，继续摚拌 $10 \mathrm{~h}$, TLC 监测反应进度 $[V$ (丙酮 $): V$ (石油醚 $)=1: 1]$. 反应 完毕, 过滤出固体, 用丙酮洗涤, 滤液在旋转蒸发器上 蒸馏得棕色粘稠状粗产物, 用乙酸乙酯重结晶, 得目标 化合物 $\mathbf{4 a} \sim \mathbf{4 h}$.

$O$-甲基-[1-(4-吗啉基)羰基亚甲基苯并咪唑-2-基]氰 基甲酮肜醚(4a), 白色固体，产率 59\%. m.p. 202.2 $202.9{ }^{\circ} \mathrm{C} ;{ }^{1} \mathrm{H}$ NMR $\left(\mathrm{CDCl}_{3}, 400 \mathrm{MHz}\right) \delta: 3.37$ (s, $2 \mathrm{H}$, Morp-H), 3.64 (s, 2H, Morp-H), $3.69 \sim 3.71(\mathrm{~m}, 4 \mathrm{H}$, Morp-H), 3.98 (s, $\left.3 \mathrm{H}, \mathrm{OCH}_{3}\right), 5.01$ [s, $\left.2 \mathrm{H}, \mathrm{NCH}_{2} \mathrm{C}(=\mathrm{O})\right]$, $7.34 \sim 7.87$ (m, 4H, Ar-H); ${ }^{13} \mathrm{C}$ NMR $\left(\mathrm{CDCl}_{3}, 100 \mathrm{MHz}\right) \delta$ : $165.1,142.6,141.5,137.0,128.0,125.6,123.6,121.3$, 110.0, 108.0, 66.7, 66.5, 45.5, 42.3; IR (KBr) v: 2238, $1667,1590,1238,1114,1028 \mathrm{~cm}^{-1}$; ESI-MS $m / z: 350.5$ $[\mathrm{M}+\mathrm{Na}]^{+}$. Anal. calcd for $\mathrm{C}_{16} \mathrm{H}_{17} \mathrm{~N}_{5} \mathrm{O}_{3}: \mathrm{C}$ 58.71, H 5.23, N 21.39; found C 58.96, H 5.02, N 21.15.

$O$-丙基-[1-(4-吗啉基)羰基亚甲基苯并咪唑-2-基]氰 基甲酮肜醚(4b)：白色固体，产率 45\%. m.p. 160.2 161.1 ${ }^{\circ} \mathrm{C} ;{ }^{1} \mathrm{H}$ NMR $\left(\mathrm{CDCl}_{3}, 400 \mathrm{MHz}\right) \delta: 0.95$ (t, $J=7.2$ $\left.\mathrm{Hz}, 3 \mathrm{H}, \mathrm{CH}_{3}\right), 1.74 \sim 1.86$ (m, $2 \mathrm{H}, \mathrm{CH}_{2} \mathrm{CH}_{3}$ ), 3.57 (d, $J=$ $4.4 \mathrm{~Hz}, 2 \mathrm{H}$, Morp-H), 3.65 (d, $J=4.8 \mathrm{~Hz}, 2 \mathrm{H}$, Morp-H), $3.69 \sim 3.74$ (m, 4H, Morp-H), 4.38 (t, $J=7.2 \mathrm{~Hz}, 2 \mathrm{H}$, $\left.\mathrm{OCH}_{2}\right), 5.09\left[\mathrm{~s}, 2 \mathrm{H}, \mathrm{NCH}_{2} \mathrm{C}(=\mathrm{O})\right], 7.32 \sim 7.91(\mathrm{~m}, 4 \mathrm{H}$, Ar-H); IR (KBr) v: 2232, 1663, 1581, 1241, 1116, 1028 $\mathrm{cm}^{-1}$; ESI-MS m/z: $378.5[\mathrm{M}+\mathrm{Na}]^{+}$. Anal. calcd for $\mathrm{C}_{18} \mathrm{H}_{21} \mathrm{~N}_{5} \mathrm{O}_{3}: \mathrm{C} 60.83$, H 5.96, N 19.71; found C $60.49, \mathrm{H}$ 6.25, N 19.82 .

$O$-丁基-[1-(4-吗啉基)羰基亚甲基苯并咪唑-2-基]氰 基甲酮肜醚(4c)：白色固体，产率 46\%. m.p. 162.0 $163.3{ }^{\circ} \mathrm{C} ;{ }^{1} \mathrm{H}$ NMR $\left(\mathrm{CDCl}_{3}, 400 \mathrm{MHz}\right) \delta: 0.96$ (t, $J=7.6$ $\left.\mathrm{Hz}, 3 \mathrm{H}, \mathrm{CH}_{3}\right), 1.38$ (q, $\left.J=7.6 \mathrm{~Hz}, 2 \mathrm{H}, \mathrm{CH}_{2} \mathrm{CH}_{3}\right), 1.72 \sim$ $1.79\left(\mathrm{~m}, 2 \mathrm{H}, \mathrm{CH}_{2} \mathrm{C}_{2} \mathrm{H}_{5}\right), 3.57$ (d, J=4.8 Hz, 2H, Morp-H), $3.65(\mathrm{~d}, J=4.8 \mathrm{~Hz}, 2 \mathrm{H}$, Morp-H), $3.69 \sim 3.74(\mathrm{~m}, 4 \mathrm{H}$, Morp-H), 4.42 (t, $J=7.6 \mathrm{~Hz}, 2 \mathrm{H}, \mathrm{OCH}_{2}$ ), $5.09[\mathrm{~s}, 2 \mathrm{H}$, $\mathrm{NCH}_{2} \mathrm{C}(=\mathrm{O})$ ], $7.32 \sim 7.92(\mathrm{~m}, 4 \mathrm{H}, \mathrm{Ar}-\mathrm{H})$; IR $(\mathrm{KBr}) v$ : 2228, 1666, 1587, 1241, 1117, $1029 \mathrm{~cm}^{-1}$; ESI-MS m/z: $392.4[\mathrm{M}+\mathrm{Na}]^{+}$. Anal. calcd for $\mathrm{C}_{19} \mathrm{H}_{23} \mathrm{~N}_{5} \mathrm{O}_{3}: \mathrm{C} 61.77, \mathrm{H}$ 6.28, N 18.96; found C 61.90, H 6.52, N 19.15.

$O$-芐基-[1-(4-吗啉基)羰基亚甲基苯并咪唑-2-基]氰 基甲酮肜醚(4d): 白色固体，产率 61\%. m.p. 135.7 $136.8{ }^{\circ} \mathrm{C} ;{ }^{1} \mathrm{H}$ NMR $\left(\mathrm{CDCl}_{3}, 400 \mathrm{MHz}\right) \delta: 3.30$ (s, $2 \mathrm{H}$, 
Morp-H), 3.53 (d, $J=4.8 \mathrm{~Hz}, 2 \mathrm{H}$, Morp-H), 3.59 3.62 (m, 4H, Morp-H), 5.07 [s, $\left.2 \mathrm{H}, \mathrm{NCH}_{2} \mathrm{C}(=\mathrm{O})\right], 5.74(\mathrm{~s}, 2 \mathrm{H}$, $\left.\mathrm{OCH}_{2}\right), 7.12 \sim 7.80(\mathrm{~m}, 9 \mathrm{H}, \mathrm{Ar}-\mathrm{H}) ;{ }^{13} \mathrm{C}$ NMR $\left(\mathrm{CDCl}_{3}, 100\right.$ MHz) $\delta: 165.0,142.2,139.8,136.5,135.0,128.4,127.4$, 126.6, 124.6, 123.1, 120.2, 112.5, 111.4, 109.4, 65.8, 65.6, 47.8, 44.0; IR (KBr) v: 2238, 1662, 1584, 1242, 1114, $1032 \mathrm{~cm}^{-1}$; ESI-MS $m / z: 426.3[\mathrm{M}+\mathrm{Na}]^{+}$. Anal. calcd for $\mathrm{C}_{22} \mathrm{H}_{21} \mathrm{~N}_{5} \mathrm{O}_{3}$ : C 65.50, H 5.25, N 17.36; found C 65.24, H 5.41, N 17.15 .

$O$-(4-氟苄基)-[1-(4-吗啉基)羰基亚甲基苯并咪唑-2基]棤基甲酮肜梄(4e): 淡黄色固体, 产率 55\%. m.p. $171.3 \sim 172.0{ }^{\circ} \mathrm{C} ;{ }^{1} \mathrm{H}$ NMR $\left(\mathrm{CDCl}_{3}, 400 \mathrm{MHz}\right) \delta: 3.46(\mathrm{~d}$, $J=4.4 \mathrm{~Hz}, 2 \mathrm{H}$, Morp-H), $3.57(\mathrm{~d}, J=4.4 \mathrm{~Hz}, 2 \mathrm{H}$, Morp-H), $3.62 \sim 3.67$ (m, 4H, Morp-H), 5.07 [s, 2H, $\mathrm{NCH}_{2} \mathrm{C}(=\mathrm{O})$ ], $5.64\left(\mathrm{~s}, 2 \mathrm{H}, \mathrm{OCH}_{2}\right), 6.95 \sim 7.97(\mathrm{~m}, 8 \mathrm{H}$, Ar-H); IR (KBr) v: 2234, 1659, 1607, 1239, 1115, 1030 $\mathrm{cm}^{-1}$; ESI-MS $\mathrm{m} / z$ : $444.1[\mathrm{M}+\mathrm{Na}]^{+}$. Anal. calcd for $\mathrm{C}_{22} \mathrm{H}_{20} \mathrm{FN}_{5} \mathrm{O}_{3}$ : C 62.70, H 4.78, N 16.62; found C 62.54, H 4.56, N 16.50.

$O$-(4-氯苄基)-[1-(4-吗啉基)羰基亚甲基苯并咪唑-2基]氰基甲酮肜醚(4f): 淡黄色晶体, 产率 59\%. m.p. 177.6 178.3 ${ }^{\circ} \mathrm{C} ;{ }^{1} \mathrm{H}$ NMR $\left(\mathrm{CDCl}_{3}, 400 \mathrm{MHz}\right) \delta$ : $3.43(\mathrm{t}$, $J=4.4 \mathrm{~Hz}, 2 \mathrm{H}$, Morp-H), $3.55(\mathrm{~d}, J=4.0 \mathrm{~Hz}, 2 \mathrm{H}$, Morp-H), $3.62 \sim 3.68(\mathrm{~m}, 4 \mathrm{H}$, Morp-H), $4.92[\mathrm{~s}, 2 \mathrm{H}$, $\left.\mathrm{NCH}_{2} \mathrm{C}(=\mathrm{O})\right], 5.64\left(\mathrm{~s}, 2 \mathrm{H}, \mathrm{OCH}_{2}\right), 6.95 \sim 7.96(\mathrm{~m}, 8 \mathrm{H}$, Ar-H); IR (KBr) v: 2237, 1657, 1610, 1236, 1112, 1030 $\mathrm{cm}^{-1}$; ESI-MS $\mathrm{m} / z: 460.5[\mathrm{M}+\mathrm{Na}]^{+}$. Anal. calcd for $\mathrm{C}_{22} \mathrm{H}_{20} \mathrm{ClN}_{5} \mathrm{O}_{3}$ : C 60.35, H 4.60, N 15.99; found C 60.58, $\mathrm{H} 4.47$, N 16.26.

$O$-(4-碘苄基)-[1-(4-吗啉基)羰基亚甲基苯并咪唑-2基]氧基甲酮肜醚 (4g)：淡黄色固体，产率 42\%. m.p. 181.2 181.9 ${ }^{\circ} \mathrm{C} ;{ }^{1} \mathrm{H}$ NMR $\left(\mathrm{CDCl}_{3}, 400 \mathrm{MHz}\right) \delta$ : 3.42 (s, $2 \mathrm{H}$, Morp-H), 3.54 (s, 2H, Morp-H), $3.62 \sim 3.66(\mathrm{~m}, 4 \mathrm{H}$, Morp-H), 4.91 [s, 2H, $\mathrm{NCH}_{2} \mathrm{C}(=\mathrm{O})$ ], $5.60\left(\mathrm{~s}, 2 \mathrm{H}, \mathrm{OCH}_{2}\right)$, 6.75 7.95 (m, 8H, Ar-H); IR (KBr) v: 2235, 1666, 1581, 1242, 1114, $1032 \mathrm{~cm}^{-1}$; ESI-MS $m / z: 552.6[\mathrm{M}+\mathrm{Na}]^{+}$. Anal. calcd for $\mathrm{C}_{22} \mathrm{H}_{20} \mathrm{IN}_{5} \mathrm{O}_{3}$ : C 49.92, H 3.81, N 13.23; found C 50.26, H 3.96, N 13.42.

$O$-(4-氧基苄基)-[1-(4-吗啉基)羰基亚甲基苯并咪 唑-2-基]氧基甲酮肜醚(4h), 白色固体, 产率 66\%. m.p. 195.6 196.4 ${ }^{\circ} \mathrm{C} ;{ }^{1} \mathrm{H}$ NMR $\left(\mathrm{CDCl}_{3}, 400 \mathrm{MHz}\right) \delta$ : $3.44(\mathrm{t}$, $J=4.4 \mathrm{~Hz}, 2 \mathrm{H}$, Morp-H), 3.56 (t, $J=4.4 \mathrm{~Hz}, 2 \mathrm{H}$, Morp-H), $3.63 \sim 3.69(\mathrm{~m}, 4 \mathrm{H}$, Morp- $\mathrm{H}), 4.90\left(\mathrm{~s}, 2 \mathrm{H}, \mathrm{NCH}_{2} \mathrm{C}(=\mathrm{O})\right.$ ), $5.73\left(\mathrm{~s}, 2 \mathrm{H}, \mathrm{OCH}_{2}\right),, 7.13 \sim 7.99(\mathrm{~m}, 8 \mathrm{H}, \mathrm{Ar}-\mathrm{H})$; IR $(\mathrm{KBr})$ v: 2225, 1657, 1607, 1227, 1093, $1030 \mathrm{~cm}^{-1}$; ESI-MS m/z:
$451.7[\mathrm{M}+\mathrm{Na}]^{+}$. Anal. calcd for $\mathrm{C}_{23} \mathrm{H}_{20} \mathrm{~N}_{6} \mathrm{O}_{3}: \mathrm{C} 64.48, \mathrm{H}$ 4.71, N 19.62; found C 64.20, H 4.93, N 19.86 .

\section{2 .2 杀菌活性试验}

采用菌丝生长速率法测定目标化合物的离体抑菌 活性 ${ }^{[21]}$. 以生菜灰霉菌(Botrytis cinerea) 和番茄菌核菌 (Sclerotinia sclerotiorum) 为测试菌种, 按培养基与药液 体积比 9:1 的比例制成含药培养基(马铃薯-葡萄糖-琼 脂, PDA), 溶解后制成浓度分别为 400、200、100、50、 $25 \mu \mathrm{g} / \mathrm{mL}$ 的 PDA 含药平板, 在培养皿中固化后将菌饼 反接到含药平板中央, 置于 $(25 \pm 1){ }^{\circ} \mathrm{C}$ 的培养箱中培养 $72 \mathrm{~h}$. 每处理设 3 个重复. 同时以无菌水为空白对照, 以 多菌灵(carbendazim) 作对照药剂. 用十字交叉法测量 菌落直径, 每个菌落测量 2 次, 以其平均值代表菌落的 大小, 按下式计算抑制率, 利用 DPS 分析软件求出测试 药剂的 $\mathrm{EC}_{50}$ 值.

$$
\begin{aligned}
& \text { 抑制百分率 }(\%)= \\
& \frac{\text { 对照菌落扩散直径一处理菌落扩散直径 }}{\text { 对照菌落扩散直径 }} \times 100 \%
\end{aligned}
$$

辅助材料(Supporting Information) 化合物 $4 \mathrm{a} \sim 4 \mathrm{~h}$ 的 ${ }^{1} \mathrm{H} N M R,{ }^{13} \mathrm{C} \mathrm{NMR}$, IR 谱图. 这些材料可以免费从本刊 网站(http://sioc-journal.cn/)上下载.

\section{References}

[1] Fu, C.; Pei, J.; Ning, Y.; Liu, M.; Shan, P.; Liu, J.; Li, Y.; Hu, F.; Zhu, Y.; Yang, H.; Zou, X. Pest Manage. Sci. 2014, 70, 1207.

[2] Dai, H.; Yu, H. B.; Liu, J. B.; Qin, X.; Wang, T. T.; Zhang, X.; Qin, Z. F.; Fang, J. X. Chin. J. Org. Chem. 2013, 33, 1104 (in Chinese). (戴红, 于海波, 刘建兵, 秦雪, 王婷婷, 张欣, 秦振芳, 方建新, 有机化学, 2013, 33, 1104.)

[3] Babazadeh-Qazijahani, M.; Badali, H.; Irannejad, H.; Afsarian, M. H.; Emami. S. Eur. J. Med. Chem. 2014, 76, 264.

[4] Jiang, L. L.; Chen, C. N.; Zhou, Y. F.; Chen, Q.; Yang, G. F. Chin. J. Org. Chem. 2009, 29, 1392 (in Chinese).

(江黎黎, 陈超南, 周延菲, 陈琼, 杨光富, 有机化学, 2009, 29, 1392.)

[5] Gannarapu, M. R.; Vasamsetti, S. B.; Punna, N.; Royya, N. K.; Pamulaparthy, S. R.; Nanubolu, J. B.; Kotamraju, S.; Banda, N. Eur. J. Med. Chem. 2014, 75, 143.

[6] Chen, Y.; Wang, Z. B.; Zhang, X.; Xia, L. J.; Gong, H. Y.; Zhao, H. J.; Xue, W. Chin. J. Org. Chem. 2014, 34, 1662 (in Chinese). (陈玉, 王忠波, 张贤, 夏丽娟, 龚华玉, 赵洪菊, 薛伟, 有机化 学, 2014, 34, 1662.)

[7] Chakravarti, B.; Akhtar, T.; Rai, B.; Yadav, M.; Siddiqui, J. A.; Dwivedi, S. K. D.; Thakur, R.; Singh, A. K.; Chattopadhyay, N.; Kumar, A. J. Med. Chem. 2014, 57, 8010.

[8] Xiao, Y.; Yan, X.; Xu, Y.; Huang, J.; Yuan, H.; Liang, X.; Zhang, J.; Wang, D. Pest Manage. Sci. 2013, 69: 814.

[9] Liu, A.; Wang, X.; Chen, C.; Pei, H.; Mao, C.; Wang, Y.; He, H.; Huang, L.; Liu, X.; Hu, Z.; Ou, X.; Huang, M.; Yao, J. Pest Manage. Sci. 2009, 65, 229.

[10] Liu, W.; He, K.; Li, Z.; Wang, X. Chemistry 2008, 71, 523 (in Chinese). 
(刘卫东, 何奇喜, 李仲英, 王晓光, 化学通报, 2008, 71, 523.)

[11] Shi, Y.; Yuan, L.; Zhang, Y.; Li, B. Chin. J. Pestic. Sci. 2007, 9, 126 (in Chinese).

(石延霞, 袁莉萍, 张一宾, 李宝聚, 农药学学报, 2007, 9, 126.)

[12] Chen, L.; Wei, N.; Gao, J.; Zhou, W. Chin. J. Pestic. Sci. 2011, 13, 448 (in Chinese).

(陈岗, 魏宁宁, 高洁, 周文明, 农药学学报, 2011, 13, 448.)

[13] Chen, X.; Yuan, H.; Qin, Z.; Qi, S.; Sun, L. Chin. J. Pestic. Sci. 2007, 9, 229 (in Chinese).

(陈小霞, 袁会珠, 覃兆海, 齐淑华, 孙丽鹏, 农药学学报, 2007, 9, 229.)

[14] Wang, M.; Qu, Z.; Du, D.; Jiang, L. Chin. J. Org. Chem. 2013, 33, 1005 (in Chinese). (王美岩，曲智强，杜丹，姜林，有机化学，2013，33, 1005.)

[15] Zhou, S.; Li, F; Zhang, P.; Jiang, L. Res. Chem. Intermed. 2013, 39, 1735.

[16] Mielczarek, M.; Thomas, R. V.; Ma, C.; Kandemir, H.; Yang X.; Bhadbhade, M.; Black, D. S.; Griffith, R.; Lewis, P. J.; Kumar, N.
Bioorg. Med. Chem. 2015, 23, 1763.

[17] Silverstein, R. M.; Bassler, G. C.; Morrill, T. C. Spectrometric Identification of Organic Compounds, 2th ed., Trans. by Yao, H.-H., Science Press, Beijing, 1988, p. 107 (in Chinese).

(西尔弗斯坦, R. M.; 巴勒斯, G. C.; 莫里尔, T. C.著, 有机化合物 光谱鉴定, (第二版), 姚海文译, 科学出版社, 北京, 1988, p. 107.)

[18] Karakurt, A.; Dalkara, S.; Özalp, M.; Özbey, S.; Kendi, E.; Stables, J. P. Eur. J. Med. Chem. 2001, 36, 421.

[19] Song, H. F.; Ding, S. M. Dyestuffs Color 2003, 40, 116 (in Chinese).

(宋华付, 丁绍民. 染料与染色, 2003, 40, 116.)

[20] Ilkun, O. T.; Archibald, S. J.; Barnes, C. L.; Gerasimchuk, N.; Biagioni, R.; Silchenko, S.; Gerasimchuk, O. A.; Nemykin, V. N. Dalton Trans. 2008, 42, 5715.

[21] Zhang, X.; Zhang, Y.; Chen, Y.; Zhou, M. Chin. J. Pestic. Sci. 2008, 10, 41 (in Chinese).

(张晓，张艳军，陈雨，周明国，农药学学报, 2008, 10, 41.) 